\title{
The Perils of Covid-19 in Nepal: Implication for Maternal Health
}

\section{Chaulagain $\mathrm{K}^{1 *}$ and Nepal $\mathrm{A}^{2}$}

${ }^{1}$ Health Emergency and Surveillance Officer, Save the Children, Nepal

${ }^{2}$ Project Coordinator, Save the Children, Nepal

*Corresponding author: Kamal Chaulagain, Health Emergency and Surveillance Officer, Save the Children, Nepal, Email: kamal.chaulagain123@gmail.com

\section{Mini Review}

Volume 3 Issue 6

Received Date: December 08, 2020

Published Date: December 24, 2020

DOI: $10.23880 /$ jqhe- 16000195

\section{Abstract}

Coronavirus (COVID-19) belongs to severe acute respiratory syndrome-corona virus-2 (SARS-Cov-2) family which is characterized as a pandemic by WHO in March 2020. With the declaration of the COVID-19 pandemic by WHO, Nepal imposed nationwide lockdown to control the transmission chain of the disease. The lockdown extended almost for 4 months which affected health system operations and management. The stress in the health system affected the access and utilization of maternal health services. As estimated by UNFPA, the pandemic could double or triple maternal deaths in Nepal by next year, due to low uptake of maternal health services such having skilled healthcare providers assist in births, delivering in health facilities, or accessing contraception. Fear of disease transmission and low trust in the maternal health services among the women, families and communities is also causes of the low uptake of the services. The COVID-19 is threatening the achievement that Nepal has acquired in maternal health outcomes in the last decade, therefore, the government need to prioritize the maternal health services during the pandemic era to avoid preventable deaths and accelerate the progress toward sustainable development goals. Appropriate interventions such as promoting hand hygiene, improve local government engagement, training and capacity building of the health workers to run the regular maternal health services, adoption of the technical guidance of the WHO for the treatment of the pregnant/postpartum women with COVID-19 symptoms/diagnosed case must be initiate by the government at each level of care. Context-specific awareness raising and behavior change communication strategy should be adopted to promote fact related COVID-19 among the community. Access to quality of care is the constitutional rights of every Nepali citizen which must be ensured at any time at any cost.

Keywords: COVID-19; WHO; ANC; United Nations

Abbreviations: COVID-19: Coronavirus; SARs-Cov-2: Syndrome-Corona Virus-2; WHO: World Health Organization; ANC: Antenatal Checkup; UN: United Nations; UNFPA: United Nations Population Fund; FCHVs: Female Community Health Volunteers; PPE: Personal Protective Equipment; GoN: Government of Nepal.

\section{Introduction}

Coronavirus disease (COVID-19) is caused by severe acute respiratory syndrome-corona virus-2 (SARS-Cov-2) a newly identified corona virus. The first case was reported in early December 2019 in the Wuhan city of China. The outbreak is later characterized as a pandemic by World Health Organization (WHO) in mid-March 2020 as result of wider epidemic in many countries [1]. The COVID-19 is primarily transmit through direct and indirect contact (through contaminated objects and surfaces) and close contact with the mouth and nose secretions released in the environment by infected person. However, there are some evidence evolving around the airborne transmission of COVID-19 which needed further research [2].

In Nepal the first case of COVID-19 was reported in $3^{\text {rd }}$ January 2020. After the declaration of the diseases as pandemic by WHO, the government of Nepal (GoN) also 
imposed nationwide lockdown to breakdown widespread transmission of disease in the community $[3,4]$. At the time there were only 2 reported positive cases. After the lock down the health services was largely affected due to supply chain disturbance, divert the local health workers in the management of the quarantine and isolation center, contact tracing, sample collection for the RDT test and PCR testing of the diseases in Nepal. This has challenged to maintain the quality of maternal health services in Nepal.

\section{Maternal, Neonatal and Child Health Situation}

The maternal morbidity and mortality in Nepal are associated with the ' 3 delays' i.e. delay in seeking care, reaching care and receiving care. In the early days of the lockdown the pregnant women were asked to hold their antenatal checkup (ANC) unless any danger sign is reported to avoid transmission from and to health facility. Similarly, due to uncertainty of the availability of health services, travel ban, difficult in accessing ambulance services etc. severely disrupted women to access maternal health care services which are critical life saving interventions for both women and child. Nepal has highest maternal mortality (239 per 100,000 live births) which indicates weak health care system. Therefore, it is predicted that the COVID-19 will further strain the health care system which would threat the achievement reached by Nepal in the maternal and neonatal health [5]. The GoN uplift the nationwide lockdown in July 21, 2020 however due to increased transmission of COVID-19 some regions have again imposed curfew to control community transmission. In the current setting of the quarantine and isolation measures, the United Nations (UN) has predicted likelihood of increased unwanted and unintended pregnancy which could escalate the risk of increased preventable maternal death [6]. The GoN has reported sharp increment in the home delivery during the COVID-19 pandemic, as only $50 \%$ of the pregnancies are now taking to the health facilities in compare with $70 \%$ before lockdown in March [6,7]. A survey conducted by the United Nations Population Fund (UNFPA) in April, found 50\% drop in the ANC visit by pregnant women which is a reason for worrying. Similarly, the UNFPA estimates pandemic could double or triple maternal deaths in Nepal by next year, as fewer women are up taking key services like: having skilled healthcare providers assist in births, delivering in health facilities, or accessing contraception [7].

Maternal stress increases among the pregnant women in the time of a pandemic. The social isolation and increased stress in pregnancy can also lead to adverse pregnancy consequences, such as preterm birth and low birth weight $[8,9]$. The social support during the pregnancy has been observed to be protective against postpartum depression and stress [10]. Many pregnant women consider delivery at home based on fear of being exposed to infection at health care setting. The evidence does not suggest intrauterine transmission of covid 19 [11]. There should be priority in treatment to pregnant women with COVID-19 because of the risk of complications. Till now, there is no evidence that pregnant women are at most risk as compared to other general population. However, an outbreak places pregnant woman at risk of infection and death [12].

\section{Way Forward}

Maternal and child health programs at Nepal are provided through peripheral level health institutions such as; birthing centers, health post and primary health care centers. Maternal, neonatal and child health should be given special attention during this pandemic situation. Country should prioritize investment in enhancing national public health capabilities and infrastructures as they are the first line of defense in public health emergencies of international concerns. Pregnant women with respiratory illnesses must be treated with greatest concern due to increased risk of adverse outcomes. The Government must segregate antenatal, neonatal and maternal health units from identified COVID-19 cases. Home visit and counseling services should be provided through female community health volunteers (FCHVs) and peripheral health workers.

One of the powerful tools to combat COVID-19 spread and transmission is promoting good hand hygiene. The regular and thorough hands washing with soap or use of hand sanitizer are critical measures to protect infection among pregnant women, children and lactating mothers. These measures should be followed by frontiers health workers. The provinces and local levels should establish and strengthen hand hygiene facilities in all peripheral health facilities.

In this pandemic situation, there are increasing death cases related to poor maternity, neonatal and inadequate child care. The government agencies should provide appropriate relief packages to the maternal mothers and children. These packages should include nutritious foods, health care supplies and sanitation related commodities. Along with relief packages, the message for breast feeding and complementary feeding including maternal nutrition must be disseminated through appropriate media.

The maternal stress coping strategies and appropriate interventions targeting pregnant and lactating mothers should be adopted in every local levels. The concept of telemedicine services for ANC checkups should be promoted. There is a need of $24 * 7$ hotline numbers for those pregnant women and lactating mothers who urgently want to seek advice and appropriate treatment. The health care providers 
must be provided with personal protective equipment (PPE) to carry out essential ANC, postnatal care and safe delivery services.

\section{Conclusion}

The Safe Motherhood programme and Reproductive Health Right Act 2018 have ensured the human rights of women and neonates in receiving health care services. During the COVID-19 pandemic, the physiological process of pregnancy and child birth neither can be stopped nor delayed. These health services must be placed to address the need of pregnant women and children. It is the responsibility of every levels of government to act on these serious issues and sustain National achievement on maternal as well as child health.

\section{References}

1. World Health Organization (WHO) (2020) Clinical management of COVID-19: Interim guidance, Geneva.

2. World Health Organization (WHO) (2020) Transmission of SARS-CoV-2: implications for infection prevention precautions, Scientific brief.

3. Ranish S, Sunit S, Pratik K, Bhvan K (2020) Nepal's first case of COVID-19 and public health response. Joirnal Travel Med 27(3).

4. (2020)Government of Nepal. Ministry of Home Affairs. Press release.

5. Aryal S, Shrestha D (2020) Motherhood in Nepal during
COVID-19 Pandemic: Are We Heading from Safe to Unsafe? Journal of Lumbini Medical College 8(1).

6. United Nations (2020) COVID-19 Nepal: Preparedness and Response Plan (NPRP).

7. (2020) The New Humanitarian. In Nepal, coronavirus drives maternal health risks as home births rise.

8. Elsenbruch S, Benson S, Rose M, Dudenhausen J, PincusKnackstedt MK, et al. (2007)Social support during pregnancy: effects on maternal depressive symptoms, smoking and pregnancy outcome. Hum Report 22(3): 869-877.

9. Shapiro GD, Fraser WD, Frasch MG, Seguin JR (2013) Psychosocial stress in pregnancy and preterm birth: Associations and mechanisms. J Perinat Med 41(6): 63145.

10. McLeish J, Redshaw M (2017) Mothers' accounts of the impact on emotional wellbeing of organised peer support in pregnancy and early parenthood: a qualitative study. BMC Pregnancy Childbirth 17: 28.

11. Chen H, Guo J, Wang C Fan L, Wei Z, et al. (2020) Clinical characteristics and intrauterine vertical transmission potential of Covid-19 infection in nine pregnant women: a retrospective review of medical records. Lancet 395(10226): 809-815.

12. Rasmussen SA, Jamieson DJ, Breese JS (2008) Pandemic influenza and pregnant women. Emerge Infect Dis 14(1): 95-100. 